\title{
Teaching Reliability at the Undergraduate Level
}

\author{
Robb Eric S. Moss, Ph.D., P.E., M.ASCE
}

\begin{abstract}
For Cal Poly's Civil and Environmental Engineering curriculum a stand-alone introductory course called Engineering Risk Analysis introduces concepts of reliability analysis. This course encompasses all the sub-disciplines in civil and environmental engineering. After five years of teaching this course there are a number of lessons that may be useful to other instructors including; solving a simple example problem using multiple methods, focusing on the normal and lognormal distributions for a quick preliminary solution, using reliability spreadsheet solutions, and allowing the students to develop their own applications of probabilistic tools. A discussion of the course format, references and resources, pedagogy devices, and inclass examples are covered. The higher goal of this course is to educate students in probabilistic methods, familiarize them with risk analysis procedures, and to elevate the basic level of understanding of uncertainty in engineering and how to properly deal with it.
\end{abstract}

\section{INTRODUCTION}

The different fields that fall under the umbrella of Civil and Environmental Engineering are reaching maturation in terms of design and analysis techniques. Almost all problems in these fields can be cast as a relationship between load and resistance or demand and capacity. The amount of data available for assessing performance of a particular design technique increases and statistical analysis can be performed. The more data that is available the more it is apparent that treating design and analysis probabilistically is the proper way to capture respective uncertainties in the load and resistance. The movement towards LRFD (Load and Resistance Factor Design) codes is because properly accounting for the uncertainty provides not just a better answer (i.e., closer to the true answer) but can also result in cost savings.

To provide graduating engineers with an understanding and appreciation of probabilistic methods I have created an upper division technical elective course called Engineering Risk Analysis that encompasses all the fields in Civil and Environmental Engineering. This course targets undergraduate students nearing the end of their degree as well as graduate students just beginning their degree. The course material is heavy on applied probabilistic methods drawing examples from Structural, 
Geotechnical, Water, Transportation, and Environmental Engineering problems to demonstrate tangible applications.

The motto for the class is "uncertainty exists, deal with it". This class allows student to become familiar with the probabilistic tools available for applied risk analysis and runs them through many examples so that they have practice tackling whatever type of problem may crop up in their own practice. This class strives for the next generation of engineers to be conversant and comfortable in probabilistic analysis.

\section{COURSE OUTLINE AND OBJECTIVES}

Engineering Risk Analysis is a ten week course (one quarter) with two 110 minute lectures a week and has the following course outline;

- Data Reduction and Sample Statistics

- $\quad$ Set Theory and Probability

- Random Variables and Probability Distributions

- Functions of Random Variables

- Reliability Analysis and Error Propagation

- Introduction to Decision Theory

- LRFD

Students are required to have completed all of their introductory courses in Structural, Geotechnical, Water, Transportation, and Environmental Engineering as well as a course in Statistics for Engineers. This has them well prepared to take a basic example from any field and examine it from a probabilistic perspective. The prerequisite statistics course covers statistical methods well and only a brief review is required, however probability is not addressed in detail in the statistics course and a substantial amount of time is spent covering this during the quarter. A thorough understanding of the fundamentals of probability is a must to progress to reliability methods.

As with most probabilists in engineering I teach this course from a Bayesian perspective. The primary reason is because failure data is often sparse, full scale failures cannot generally be duplicated in a controlled manner, and sample sets are highly heterogeneous and non-ergodic. Subjective probability is often needed to augment the meager objective probability measures available. The subjective probability can be from a prior information, expert consensus, and engineering judgment. Philosophically this follows the degree-of-belief definition of probability which is consistent with the design process in Civil and Environmental Engineering problems and is also consistent with hazard forecasting and hazard mitigation.

In this course we work primarily the normal and lognormal probability distributions. This simplifies the mathematics greatly in a number of areas and provides a reasonable first-order approximation for most engineering problems. References for other theoretical distributions are many and if students understand the basis for using a theoretical distribution they can often easy increase the complexity of the analysis by using other distributions once they have run through it with the normal or lognormal.

The guiding principle and objective of this course; all undergraduate engineering 
students should be able to perform simple reliability analysis. By presenting reliability in a simplified format not only do the students gain the ability to evaluate load versus resistance in a probabilistic manner but more importantly they leave the class with a new perspective on engineering. This perspective is that uncertainty exists in every aspect of engineering and that they must deal with it either implicitly or explicitly. For most students this is a subtle but profound paradigm shift that alters the way they view all subsequent engineering problems.

The class culminates in a self-directed project where the students are asked to take some of their newly acquired tools and apply them to an engineering problem of their choosing. The project is purposely left open ended to allow for exploration of topics and tools. The projects do not necessarily have to use reliability methods but the better students often gravitate towards this because they find it a powerful extension of their engineering abilities. Examples of successful student projects are presented later in this manuscript.

\section{RESOURCES}

There are a number of excellent resources that I have drawn heavily from in developing this course, although no single text or reference suffices. The discussion of statistics draws from the venerable Benjamin and Cornell (1970) which is dated but I find does the best job presenting the simple applied statistics needed for the course. Discussion of probability, set theory, probability distributions, functions of random variables, and Monte Carlo simulations closely follows the excellent revised text by Ang and Tang (2007). The first edition of this text is what most current engineers "cut their teeth on". While that was a good text, it was often hard to navigate and opaque on some topics. The second edition of this text is far superior, taking the strong points of the first edition (Ang and Tang 1975) and adding to it better explanations, more and better examples, and computation solutions; the way most problems are solved in this era of inexpensive computation effort.

Once through the topic of functions of random variables by mid-quarter, enough ground has been covered to move into reliability. Here the class starts with Baecher and Christian (2003) which I find provides the most clear and concise review of reliability methods. Other references have been drawn from including Ang and Tang (1990), Der Kiureghian (2001), and Stewart and Melchers (1997).

After spending a fair amount of lecture time on component reliability the class ventures into systems analysis, decision trees, and broader risk topics. Here along with material drawn from the references mentioned above a number of examples are drawn from my own research and consulting experience. I find it useful to bring in an external speaker who does environmental risk analysis to present a couple complex projects utilizing the tools we have covered in a basic manner in class.

With reliability behind us we can now discuss the basis of LRFD codes and how the factors are developed. I find the presentation by Baecher and Christian (2003) is accessible to the students and the given examples show the utility of LRFD over WSD/ASD (working stress design/allowable stress design. 


\section{EXAMPLE PROBLEMS}

The most useful example is one that I borrow from Baecher and Christian (2003). Here my bias for geotechnical engineering is obvious, but the "cut slope" problem is so simple and accessible to students that it is hard to justify using another. The problem deals with a vertical cut in cohesive soil. Figure 1 shows the geometry and details of the problem.

As mentioned previously students in the class are required to complete all their introductory engineering courses so they have had an introduction to geotechnical engineering and are familiar with soil mechanics. This problem is used to explore a suite of reliability solution techniques; exact solution(s), first order second moment (FOSM), first order reliability method (FORM), second order reliability method (SORM), and Monte Carlo Simulations. With FORM and SORM, whereas solutions following the improved Hasofer-Lind algorithm (Zhang and Der Kiureghian 1995) are presented for completeness, the focus is on simplified spreadsheets solutions (Low and Tang 1997; Phoon 2008; Phoon and Nadim 2004) because of their accessibility to students with a limited advanced mathematics background. The "cut slope" problem reinforces the concepts covered when discussing functions of random variables. It is also useful for exploring how correlation between independent variables can influence the component reliability.

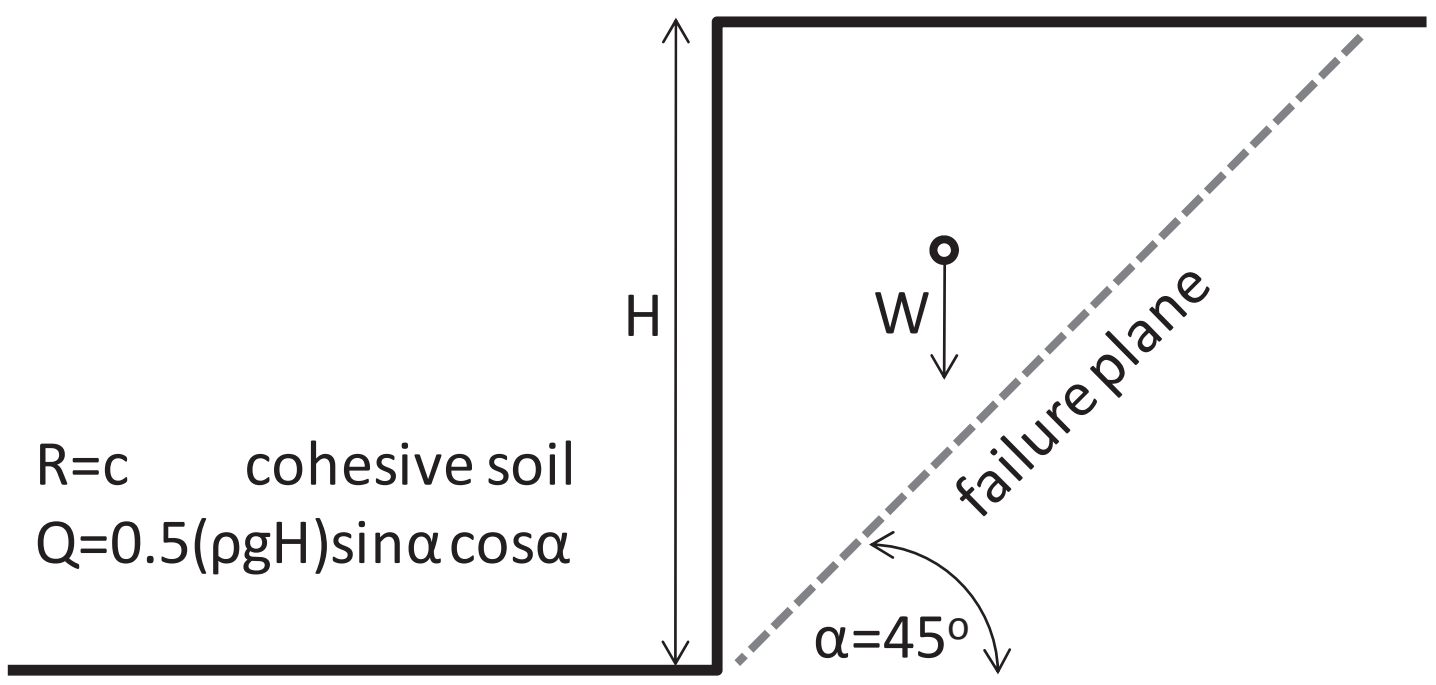

FIG. 1. Cut slope problem used to demonstrate exact, FOSM, FORM, SORM, and MC reliability solution methods. In this figure $H$ is the vertical cut height, $W$ the weight at the centroid of the soil mass above the failure plane, alpha $(\alpha)$ the failure plane angle, $R$ the resistance which is equal to the soil cohesion (c), and $Q$ the load which is the product function shown that includes density ( $\rho)$ and gravity (g). 


\section{PEDAGOGICAL TECHNIQUES}

After teaching this course for five years I have found some pedagogical techniques that are useful. Often the hurdle in this class is getting the students through the section on elementary probability. After covering Venn diagrams, set theory, and the rules of probability we spend the bulk of two lectures going through example problems. The trick with problems of elementary probability is that there are often different means of solving them, whether using a prior probability, Venn diagrams, and/or invoking the rules of probability. But the most difficult aspect of elementary probability problems is taking a word statement and writing it correctly in set theory with the appropriate probability terms and set theory symbology. To help the students a simple rubric is repeated over and over in problem solving;

a) Declare all knowns (an obvious but often overlooked step),

b) Write the problem statement in set terminology (this takes much practice), and

c) Reduce the stated to a calculatable form by invoking the rules of probability (often the easiest step).

In this section of the class I assign a set of homework and ask the students to work in pairs. I encourage group discussions among pairs and forewarn them that these problems, although they appear simple, often require a good deal of time to work through. The day the homework is handed in I often take lecture time to go through the problems one by one, show them my solution to the problem, and have an in class discussion. The written solutions to these problems are provided with clear step by step declarations following the above rubric and explicitly stating how the steps were arrived at. I have found that this careful and thorough coverage is necessary to assist the students through the crux of probability and lays the foundation for the reliability section to come.

A second pedagogical technique that is used is restricting our use of probability distributions to the normal and lognormal. This provides mathematical simplicity in almost all cases and allows for the concepts of probability and reliability to be focused on rather than dwelling on the menagerie of probability distributions and their nuances. This allows the class to progress through reliability in one quarter and gives the students a means of performing a first-order estimate of reliability for the bulk of engineering problems. There are many texts and other references that exist on other theoretical probability distributions and I provide some discussion where it may be necessary to diverge from the normal and lognormal. But for most applications the normal and lognormal are sufficient to give a close enough approximation for firstorder engineering assessment.

\section{STUDENT PROJECTS}

The quarter culminates in students picking a particular topic of interest from their discipline and applying some of the tools they acquired during the course. The student project deliverables are a short written report and a short oral presentation. This is often the most rewarding portion of the course from an instructor's perspective. Some of the more interesting student projects over the years include;

- A student was interested in power distribution systems and used a recent journal publication to develop uni-, bi-, and tri-modal estimates of systems 
failure for a particular grid arrangement with a specific correlation structure.

- A structural student was interested in reliability of timber beams for home construction. There was some statistical information in the literature on the strength properties of timber beams but the student decided to augment the sample statistics by collecting over a hundred popsicle sticks and individually testing them to failure to better characterize the variance. The mean results when scaled were in good agreement with the published mean results, and the variance (and frequency distribution) was much better characterized thanks to the experiments.

- A traffic student was interested in the probability of cars stopping for "right on red" situation and, finding no data available, spent several hours at a local corner collecting data to inform the analysis. The presentation was rather lively as the discussion included scores of samples where cars were not stopping at the light at all regardless of pedestrians (including babies in strollers) in the crosswalk.

Typical projects include error propagation through common engineering design equations (e.g., open channel flow, reinforced concrete deign, waste water discharge concentration), or statistical analysis with an elementary probability statement at the end (e.g., traffic flow, construction cost analysis). But there are a number of these projects that are developed enough to end up as chapters in master's theses or incorporated into research reports. In this sense the class is a resource for the department in providing a probabilistic perspective for any student in Civil and Environmental Engineering looking to develop that aspect of their research. The key to the student projects is that they are open ended with the topic driven by the student's interest, resulting in a more intimate mastery of some of the tool introduced in the class.

\section{SUMMARY}

Presented is a discussion of a course positioned in Cal Poly's Civil and Environmental Engineering curriculum that introduces reliability to upper division undergraduates, as well as first year graduate students. My goal in creating this course was to emphasize applied statistics and probability to enable the students to perform simple first-order estimates of the probability of failure for any problem they may encounter.

To achieve this the fundamentals are covered in a purposeful, detailed, and applied manner so that the students master sample statistics, elementary probability, simple probability distributions (normal and lognormal), functions of random variables, component reliability, and systems reliability. This requires a large number of in class examples that follow a problem solving rubric and show clear step by step solutions. The class culminates in a student directed project on a topic of their choosing where the tools developed in the class are applied.

Success in developing this class is demonstrated by the large number of student who go on to incorporate probabilistic methods into their engineering thought process and carry that over into their practice or research. This class strives to imbue the next generation of engineers with an appreciation of uncertainty in Civil and 
Environmental Engineering problems and to arm them with tools for dealing with this uncertainty in an explicit manner.

\section{REFERENCES}

Ang, A. H.-S., and Tang, W. H. (1975). Probability Concepts in Engineering Planning and Design: Volume I. Basic Principles. John Wiley and Sons, Inc.

Ang, A. H.-S., and Tang, W. H. (1990). Probability Concepts in Engineering Planning and Design: Volume II-Decision, Risk, and Reliability, Published by the Authors.

Ang, A. H.-S., and Tang, W. H. (2007). Probability Concepts in Engineering: Emphasis on Applications to Civil and Environmental Engineering. 2nd Edition. John Wiley and Sons, Inc., New York.

Baecher, G. B., and Christian, J. T. (2003). Reliability and Statistics in Geotechnical Engineering, John Wiley and Sons, Inc.

Benjamin, J., and Cornell, C. A. (1970). Probability, Statistics, and Decision for Civil Engineers, McGraw-Hill, New York.

Der Kiureghian, A. (2001). "Course Notes from CE229: Structural Reliability." UC Berkeley.

Low, B. K., and Tang, W. H. (1997). "Efficient Reliability Evaluation Using Spreadsheet." Journal of Engineering Mechanics, 123(7), 749-752.

Phoon, K.-K. (2008). "Reliability-Based Design in Geotechnical Engineering." Taylor and Francis, New York.

Phoon, K.-K., and Nadim, F. (2004). "Modeling Non-Gaussian Ramom Vectors for FORM: State-of-the-Art Review (keynote)." International Workshop on Risk Assessment in Site Characterization and Geotechnical Design, Indian Institute of Science, Bangalore, India, 55-85, November 26-27.

Stewart, M. G., and Melchers, R. E. (1997). Probability risk assessment of engineering systems, Chapman\&Hall.

Zhang, Y., and Der Kiureghian, A. (1995). "Two improved algorithms for reliability analysis." Proceedings 6th IFIP WG 7.5 working conference on reliability and optimization of structural systems, 297-304, 